\title{
Zentralvorstandssitzung vom 16. September 2010
}

TARMED/Beibericht Evaluation EFK - Die FMH verfasst einen Beibericht zum Schlussbericht «Evaluation TARMED» der eidgenössischen Finanzkontrolle (EFK). Sie tritt damit deren Empfehlungen entgegen, nach denen der Bund mehr Einfluss auf TARMED erhalten soll.

Revision Analysenliste - Der Zentralvorstand der FMH fordert aufgrund der Resultate, dass der Übergangsfranken per Ende 2011 nicht gestrichen und eine separate Berechnung für Praxislabors eingeführt wird (Point of care).

Besuchsinkonvenienz-Pauschale (BIP) - Die FMH beantragt eine Verlängerung der BIP bis Ende Februar 2012.

Ärztliche Medikamentenabgabe (AMA) - Die FMH wird mit santésuisse über die margenfreie Medikamentenabgabe verhandeln. Vorgesehen ist eine Umlagerung auf den TARMED.

6. IV-Revision - Der ZV nimmt Stellung zum zweiten Massnahmenpaket (Revision 6b) und fokussiert dabei auf jene Punkte der Vorlage, die einen Bezug zur Medizin haben, wie interprofessionelles Assessment, Eingliederungsfähigkeit oder zumutbare medizinische Massnahmen.
Ärztliche Qualitätsarbeit - Der ZV stimmt zwei Grundlagenpapieren $\mathrm{zu}$, die folgende Themen umreissen:

- Die Fachgesellschaften haben die Kompetenz festzuhalten, welchen Aufwand und Nutzen die ärztlichen Qualitätsaktivitäten in ihrem Fachgebiet haben; die FMH unterstützt sie dabei.

- Ärztliche Qualitätsarbeit ist zu unterstützen und zu fördern, vor allem mit nicht-monetären Anreizen.

Kindsmisshandlung - Die FMH hat das Vorwort des Leitfadens für Ärztinnen und Ärzte zur Früherfassung von Gewalt an Kleinkindern der Stiftung Kinderschutz Schweiz verfasst und das FMH-Logo dafür zur Verfügung gestellt.

Unverjährbarkeits-Initiative - Der Zentralvorstand unterstützt die Initiative, dass gemäss Straf- und Militärstrafgesetz sexuelle und pornographische Straftaten an Kindern vor der Pubertät unverjährbar werden sollen. Er konzentriert sich dabei auf die Beweisschwierigkeiten und äussert sich nicht zur Altersgrenze.

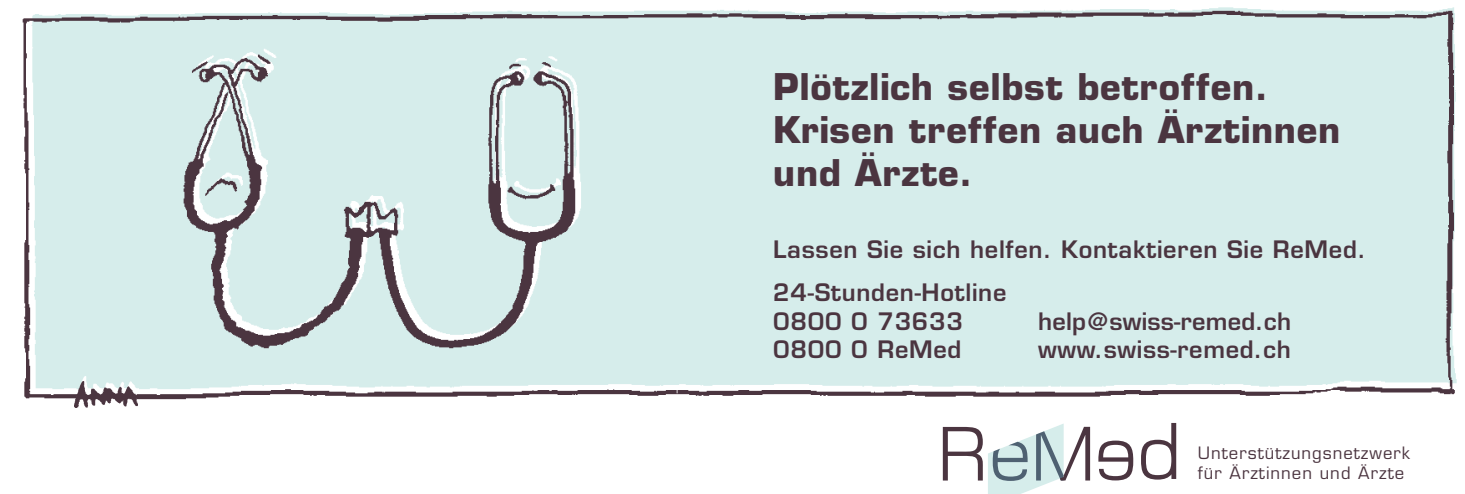

